\title{
Chromium removal from aqueous media by superparamagnetic starch functionalized maghemite nanoparticles
}

\author{
P N SINGH, D TIWARY and I SINHA* \\ Department of Chemistry, Indian Institute of Technology (Banaras Hindu University), Varanasi 221 005, India \\ e-mail: isinha.apc@itbhu.ac.in
}

MS received 22 February 2015; revised 14 August 2015; accepted 18 August 2015

\begin{abstract}
Superparamagnetic starch functionalized maghemite nanoparticles (SMhNPs) were synthesised by a co-precipitation method via in situ functionalization and used as nano-adsorbents for the removal of $\mathrm{Cr}(\mathrm{VI})$ from aqueous waste. The characterization of the prepared nanoparticles was done by XRD, FTIR, TEM and VSM techniques. Adsorption of $\mathrm{Cr}(\mathrm{VI})$ on the surface of superparamagnetic starch functionalized maghemite nano-adsorbents was investigated and the removal was higher in acidic $\mathrm{pH}$ as compared to that exhibited in basic medium. The adsorption of $\mathrm{Cr}(\mathrm{VI})$ by the SMhNPs followed pseudo-second order kinetics and the adsorption isotherm data fits well the Freundlich adsorption isotherm. The $K_{F}$ value for $\mathrm{Cr}(\mathrm{VI})$ removal by SMhNPs is found to be $24.76 \mathrm{mg} . \mathrm{g}^{-1}$, which is significantly better than the adsorption capacities reported in literature for maghemite nanoparticles. Thermodynamic studies revealed that the adsorption of $\mathrm{Cr}(\mathrm{VI})$ onto the superparamagnetic starch functionalized maghemite nano-adsorbents is spontaneous and endothermic in nature.
\end{abstract}

Keywords. Chromium removal; starch; maghemite nanoparticles; adsorption.

\section{Introduction}

Industrial wastewater often contains substantial amount of hexavalent chromium mainly from rinsing of electroplated articles, drag out from the chromium bath and spent chromate passivation solutions. Such effluents pose severe threat to public health and the environment if discharged without adequate treatment. ${ }^{1}$ Hexavalent chromium is therefore mobile in the environment. Further, $\mathrm{Cr}(\mathrm{VI})$ can easily penetrate the cell wall and hence causes cancer and other fatal ailments. Thus, highly toxic hexavalent chromium species act as carcinogens, mutagens and teratogens in biological systems. ${ }^{2}$ Therefore, USEPA has set the permissible limit of chromium in water at $<0.1 \mathrm{mg} / \mathrm{L}$ level. ${ }^{3}$ In view of the above discussion, it is imperative that industries reduce chromium in their effluents to this acceptable level before discharging into municipal sewers. A variety of methods have been developed and used for the removal of chromium compounds from industrial wastewater.

In nature, chromium exists in two most common oxidation states, viz. $\mathrm{Cr}(\mathrm{III})$ and $\mathrm{Cr}(\mathrm{VI})$. $\mathrm{Cr}(\mathrm{VI})$ oxidation state is more ubiquitous and toxic in comparison to $\mathrm{Cr}(\mathrm{III})$ oxidation state. ${ }^{4,5} \mathrm{Cr}(\mathrm{VI})$ may be reduced and converted into the less toxic and immobilized $\mathrm{Cr}$ (III) oxidation state. ${ }^{6}$ Hexavalent chromium usually exists

\footnotetext{
*For correspondence
}

in wastewater as oxyanions such as chromate $\left(\mathrm{CrO}_{4}^{2-}\right)$ and dichromate $\left(\mathrm{Cr}_{2} \mathrm{O}_{7}^{2-}\right)$, and does not precipitate easily by conventional methods. Besides reduction, many different treatment methods have been developed for the remediation of $\mathrm{Cr}(\mathrm{VI})$ like reverse osmosis, electro dialysis, ion exchange and adsorption. Among these, adsorption is an efficient and cheap technique available for wastewater treatment. ${ }^{7,8}$ Different natural adsorbents have been tried for the removal of $\mathrm{Cr}(\mathrm{VI})$ like wool, olive cake, sawdust, pine needles, almond shells, cactus leaves, charcoal of used tyres, soot, hazelnut shell, coconut shell charcoal, banana peel, seaweed, dead fungal biomass, cyanobacteria, green alga, etc. ${ }^{9-15}$ However, most of these adsorbents have low adsorption capacity for $\mathrm{Cr}(\mathrm{VI})$ removal. Consequently, there is a need for development of new environmentally friendly or green adsorbents having larger surface area and better durability.

In this respect, magnetic iron and iron oxide nanoparticles are important adsorbents for cleanup of heavy metal contaminants like $\mathrm{Cr}(\mathrm{VI})$ because of their small particle size, high surface area, low cost, and ease of preparation. ${ }^{16-19}$ Earlier studies by $\mathrm{Hu}$ et al. have already demonstrated good adsorption capacity of magnetite and maghemite nanoadsorbents for $\mathrm{Cr}(\mathrm{VI})$ from aqueous medium. ${ }^{20}$ If the nanoparticle size is fine enough then the nanoadsorbents would be superparamagnetic. Hence, this adsorbent could be easily separated 
from the water by using external magnets. Superparamagnetic nature of such nanoadsorbents significantly enhances the ease of redispersion and the repeated reuse of these nanoadsorbents. Accordingly, in the present study we focus on use of superparamagnetic functionalized maghemite nanoparticles as adsorbents for $\mathrm{Cr}(\mathrm{VI})$ from aqueous medium. The emphasis is on the effect of surface functionalization of magnetic nanoparticles on their adsorption properties. Surface protecting agents or stabilizers prevent or minimize aggregation which enhances the availability of surface active sites on the adsorbent. The objective of such surface protection could be twofold, that is, not only to stabilize the maghemite nanoparticles, but also to provide further functionalization of the surface which can enhance or modify properties of nanoparticles. ${ }^{12}$ Recently, many researchers have used water soluble starch for nanoparticle functionalization or stabilization. Starch is an environmentally green low-cost natural biocompatible polymer. The hydrophilic and biodegradable nature of water soluble starch further enhances possibilities with respect to functionalization and also helps in increasing the dispersibility of these nanoparticles in water. Owing to biocompatible and biodegradable nature of starch, such functionalized nanoparticles could also be used in drug delivery systems. ${ }^{21}$ In this communication, we first present the synthesis of superparamagnetic starch functionalized maghemite nanoparticles (SMhNPs) and then use them as nano adsorbents for the removal of $\mathrm{Cr}(\mathrm{VI})$ from aqueous medium. We would also like to mention here that to the best of our knowledge this is the first report of such synthesis of superparamagnetic SMhNPs. The stress is on the investigation of the removal of $\mathrm{Cr}(\mathrm{VI})$ by superparamagnetic SMhNPs. We report and discuss both kinetic and thermodynamic parameters to understand the mechanism of $\mathrm{Cr}(\mathrm{VI})$ adsorption onto the surface of superparamagnetic SMhNPs.

\section{Experimental}

\subsection{Synthesis of SMhNPs adsorbents}

Chemicals used were ferrous sulphate heptahydrate (Merck), anhydrous ferric chloride (Merck), sodium hydroxide (Merck) and starch (Merck). All chemicals were of analytical grade and used without further purification. The following co-precipitation protocol was used for synthesis of SMhNPs. Required amounts of $\mathrm{FeCl}_{2}$ and $\mathrm{FeCl}_{3}$ salts in molar ratio of 1:2 were dissolved in $250 \mathrm{~mL}$ of distilled water and mechanically stirred for 15 min. Next 2 wt $\%$ of freshly prepared aqueous starch solution was added to the previously prepared solution with continuous mechanical stirring for about $30 \mathrm{~min}$.
This mixed solution was added drop wise into previously heated $\mathrm{NaOH}$ solution $(0.8 \mathrm{M})$ maintained at $80^{\circ} \mathrm{C}$. The whole reaction mixture was heated at $80^{\circ} \mathrm{C}$ for two more hours until dark brown precipitate formation was observed. The $\mathrm{pH}$ of the reaction mixture was found to be $>12$. The precipitate was separated by decantation using an external magnet and then repeatedly washed with distilled water in order to remove excess $\mathrm{NaOH}$ and finally washed with absolute alcohol to make it neutral. We also measured the $\mathrm{pH}$ of the filtrate using $\mathrm{pH}$ meter (Systronic $\mu$ system 361) and found it to be neutral. The precipitate was then dried at $40^{\circ} \mathrm{C}$ in a hot air oven. Now the dried powder samples were ground and used for further adsorption studies. The sample for TEM imaging was prepared in the following way. A small amount of the powder sample was redispersed in $30 \mathrm{~mL}$ of deionized water and sonicated (Lobachemie ultrasonic cleaner at $42 \mathrm{kHz}$ and $170 \mathrm{~W}$ ) for about $10 \mathrm{~min}$. Then a small volume of the aqueous colloidal suspension thus obtained was further diluted by a fixed factor and again sonicated to minimize van der Waals interactions. In the final step a drop of this diluted sol was deposited on to the carbon-coated copper grid and dried in a desiccator.

\subsection{Batch Adsorption Experiment}

The stock solution of $\mathrm{Cr}(\mathrm{VI})$ was prepared by dissolving known amount potassium dichromate in distilled water. Solution $\mathrm{pH}$ was adjusted by adding required amounts of $0.1 \mathrm{M} \mathrm{HCl}$ or $0.1 \mathrm{M} \mathrm{NaOH}$. In all adsorption experiments $4 \mathrm{~g} . \mathrm{L}^{-1}$ of the adsorbent was added into aqueous $\mathrm{Cr}(\mathrm{VI})$ solutions of different initial concentrations in stoppered conical flasks. These solutions were then subjected to agitation for $24 \mathrm{~h}$ on a thermostatic shaker (Narang Scientific), which was more than sufficient for attainment of equilibrium. ${ }^{22}$ The adsorbents in these solutions were then separated from the $\mathrm{Cr}(\mathrm{VI})$ solution by decantation using an external magnet. Now the residual concentration of $\mathrm{Cr}(\mathrm{VI})$ was measured by UV-Visible spectroscopy (Perkin Elmer) at wavelength $540 \mathrm{~nm}$ using 1,5-diphenylcarbazide (Merck) method.

The amount of $\mathrm{Cr}(\mathrm{VI})$ adsorbed by the nano adsorbents at equilibrium $\left(q_{e}, \mathrm{mg} \cdot \mathrm{g}^{-1}\right)$ per unit mass of the adsorbent was calculated by equation 1 .

$$
q=\left(C_{o}-C_{e}\right) \times \frac{V}{m}
$$

Here, $C_{o}$ and $C_{e}$ are the initial and equilibrium concentrations of $\mathrm{Cr}(\mathrm{VI})$ solution $\left(\mathrm{mg} . \mathrm{L}^{-1}\right)$ respectively. Further, $V$ denotes the volume of the solution taken in litres and $m$ is the mass of adsorbent in grams. 
Equation 2 is used to calculate the adsorption percentage $(\% A d)$ of $\mathrm{Cr}(\mathrm{VI})$ ions.

$$
\% A d=\frac{\left(C_{o}-C_{e}\right)}{C_{o}} \times 100
$$

\section{Results and discussion}

\subsection{Characterisation of SMhNPs}

XRD data of the prepared SMhNPs adsorbent (figure 1) were collected from 2 theta range 10 to $90^{\circ}$. The planes $311,440,511,220$ and 400 match very well with the maghemite phase and no other phase could be detected, which indicates that the product formed is highly pure and crystalline in nature. ${ }^{23}$ The FTIR spectra of starch and SMhNPs are shown in figures 2(a) and 2(b), respectively. The FTIR spectra of starch shows the fundamental peaks at $1171,1019 \mathrm{~cm}^{-1}$ due to prominent C-O-C stretching vibration of glycosides bonds and stretching vibration of $\mathrm{C}-\mathrm{O}$ bonds, respectively. Normally the stretching frequency of $\mathrm{Fe}-\mathrm{O}$ bonds peaks is observed at about $582 \mathrm{~cm}^{-1} \cdot{ }^{21,24,25}$ Owing to chemisorption of starch on to the surface of the maghemite nanoparticles, this peak shifts to $616 \mathrm{~cm}^{-1}$ in the FTIR spectrum of SMhNPs. This is in addition to the starch fundamental peaks already present in the SMhNPs FTIR spectrum. Figure 3 displays the Raman spectrum of the starch functionalized maghemite nanoparticles. The Raman spectrum does show peaks at 350,500 and $702 \mathrm{~cm}^{-1}$ which are due to the maghemite phase. ${ }^{26-28}$ Furthermore, peaks are also present at 479 and $673 \mathrm{~cm}^{-1}$.
The appearance of these peaks may be assigned to the presence of skeletal modes of pyranose ring of starch molecules. Peaks at 1087 and $1122 \mathrm{~cm}^{-1}$ correspond to $\mathrm{C}-\mathrm{O}-\mathrm{H}$ bending and $\mathrm{C}-\mathrm{O}$ stretching mode of glycosidic skeleton of starch molecule, respectively. On combining this information with the FTIR spectrum analysis, we conclude that starch is chemisorbed on the surface of maghemite nanoparticles.

Figure 4(a) displays TEM images of typically spherical SMhNPs formed. All nanoparticles are found to be well separated. Particle size distribution obtained from TEM images of SMhNPs using ImageJ analysis software are given in figure 4(b). Fine particle size distribution in the range of $4-17 \mathrm{~nm}$ is obtained. In order to investigate the magnetic properties of SMhNPs, vibrating sample magnetometer (VSM) measurements were carried out. VSM plots of magnetization verse magnetic field (M-H loop) at room temperature for SMhNPs is shown in figure 5 . Saturation magnetization $\left(M_{\mathrm{s}}\right)$, remanent magnetization $\left(M_{\mathrm{r}}\right)$ and Coercivity $\left(H_{\mathrm{c}}\right)$ of starch functionalized maghemite nanoparticles were found to be $17.57 \mathrm{emu} / \mathrm{g}, 0.000014802 \mathrm{emu} / \mathrm{g}$ and 0.03337 gauss, respectively. Low $M_{\mathrm{r}}$ and $H_{\mathrm{c}}$ values indicate that the SMhNPs are superparamagnetic in nature.

\subsection{Effect of $p H$ on adsorption properties}

The $\mathrm{pH}$ of the adsorbate solution is an important parameter that affects the adsorption capacity of the SMhNPs. It not only changes surface charge density of adsorbent but also the degree of ionization and speciation of

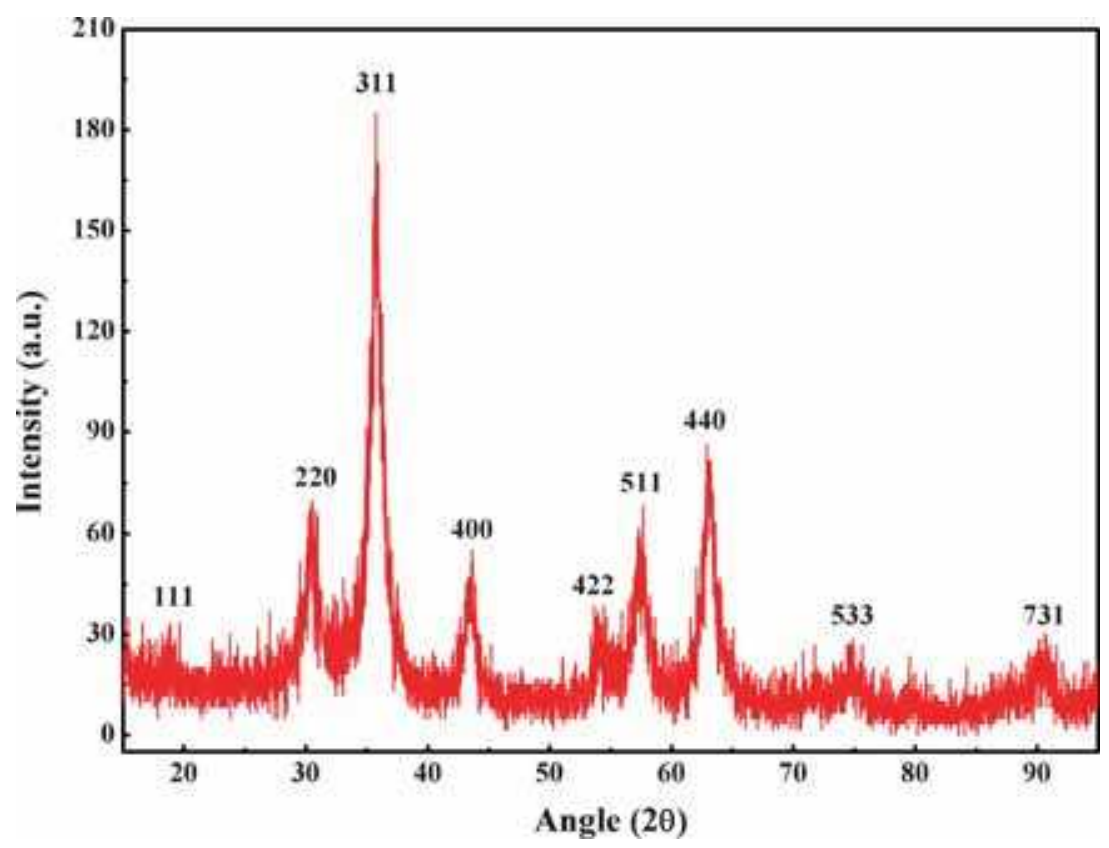

Figure 1. XRD pattern of SMhNPs adsorbent sample. 


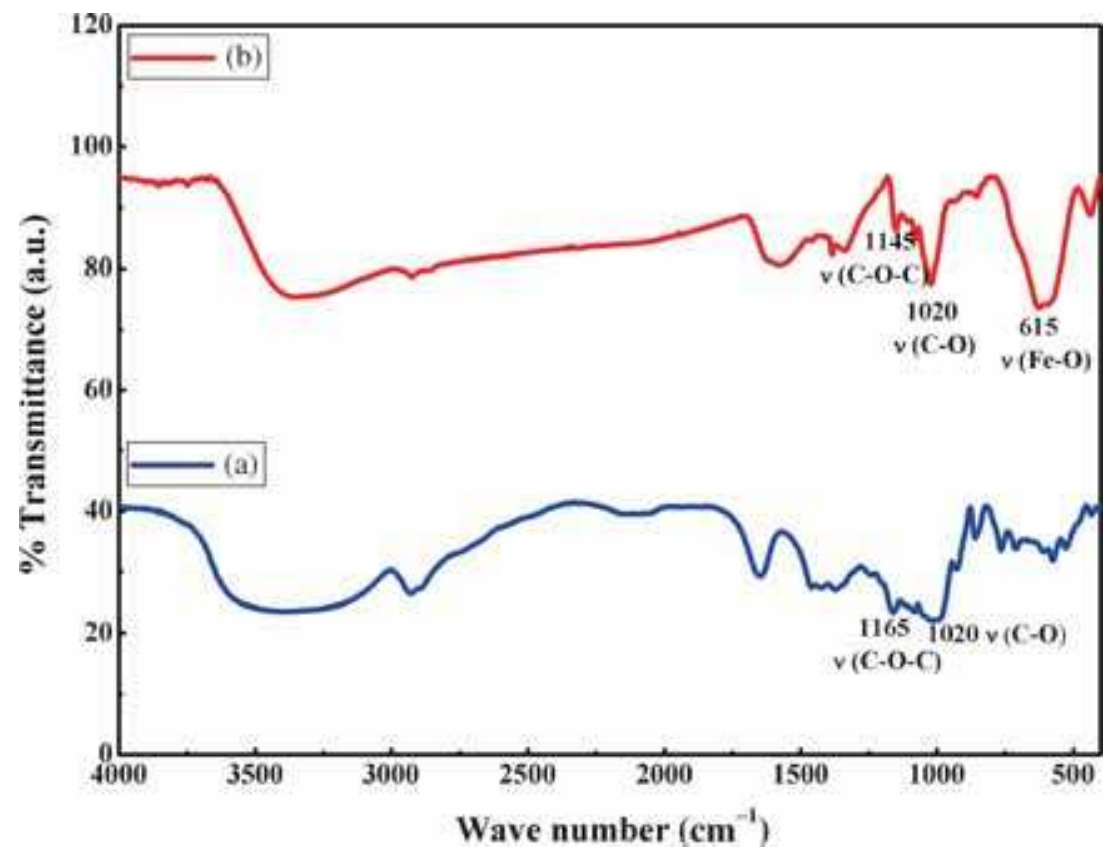

Figure 2. Comparative FTIR plots of (a) Starch; and (b) SMhNPs demonstrating starch functionalization of nanoparticles.

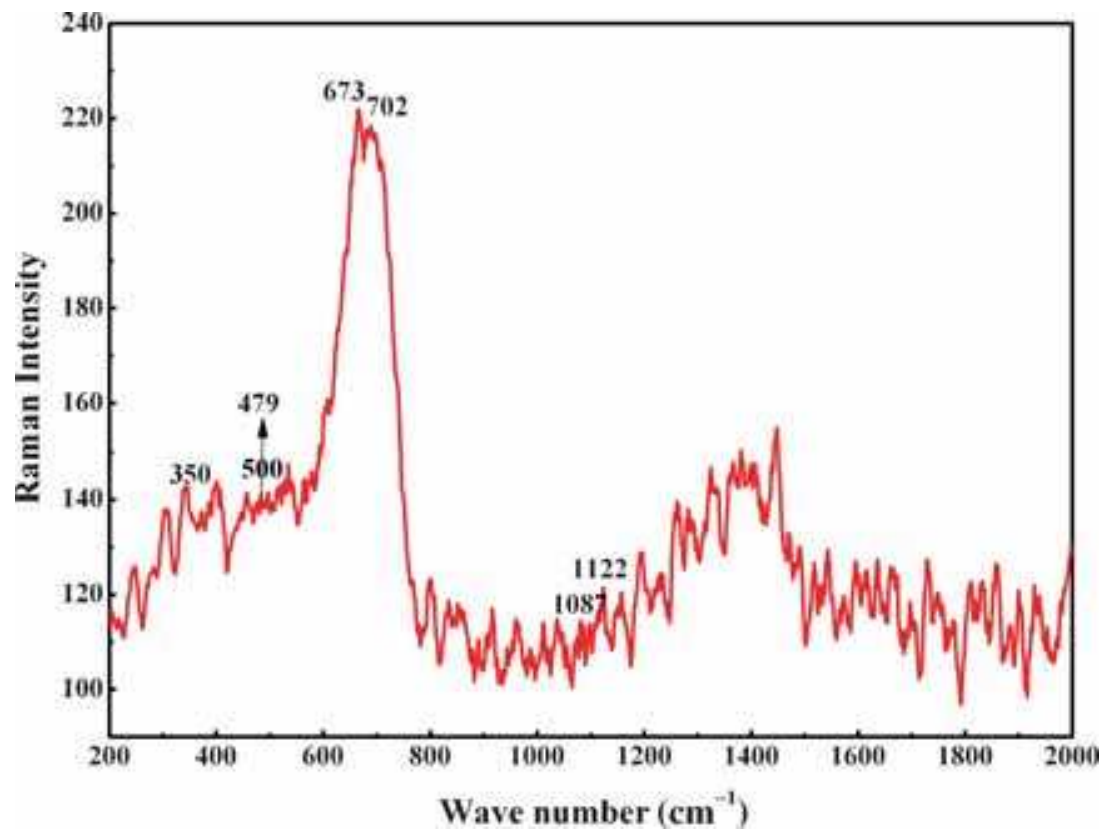

Figure 3. Raman spectrum of the starch functionalized maghemite nanoparticles.

adsorbate during the adsorption reactions. Adsorption capacity was higher in acidic $\mathrm{pH}$ range and decreased with increase in $\mathrm{pH}$. The adsorption capacity versus $\mathrm{pH}$ plot (figure 6a) shows that the optimum removal $\left(q_{\mathrm{e}} \sim 9.8 \mathrm{mg} \cdot \mathrm{g}^{-1}\right)$ of $\mathrm{Cr}(\mathrm{VI})$ by s SMhNPs occurs at $\mathrm{pH}$ 2 and $303 \mathrm{~K}$. Thereafter it decreases and gradually falls to $q_{\mathrm{e}}<8 \mathrm{mg} . \mathrm{g}^{-1}$ at $\mathrm{pH} 8$. In order to get a better understanding of the role of $\mathrm{pH}$, the point of zero charge of the new adsorbent (SMhNPs) was determined (figure $6 \mathrm{~b}$ ). Point of zero charge $\left(\mathrm{pH}_{\mathrm{PZC}}\right)$ of the starch functionalized maghemite nano-adsorbents was found to be 7.95. While the adsorbent surface is neutral at the $\mathrm{pH}_{\mathrm{PZC}}$, the adsorbent surface is positively charged when the $\mathrm{pH}$ of the solution is less than $\mathrm{pH}_{\mathrm{PZC}}$ and negatively charged when $\mathrm{pH}>\mathrm{pH}_{\mathrm{PZC}}$. The reason for the high adsorption capacity in acidic reason may be due to the relatively higher positive charge density at lower $\mathrm{pH}$ on the adsorbent surface. The species of $\mathrm{Cr}(\mathrm{VI})$ present depends on the $\mathrm{pH}$ of the solution. Thus, $\mathrm{Cr}(\mathrm{VI})$ exists as $\mathrm{HCrO}_{4}^{-}$ in acidic $\mathrm{pH}$, as $\mathrm{Cr}_{2} \mathrm{O}_{7}^{2-}$ in neutral $\mathrm{pH}$ and as $\mathrm{CrO}_{4}^{2-}$ 


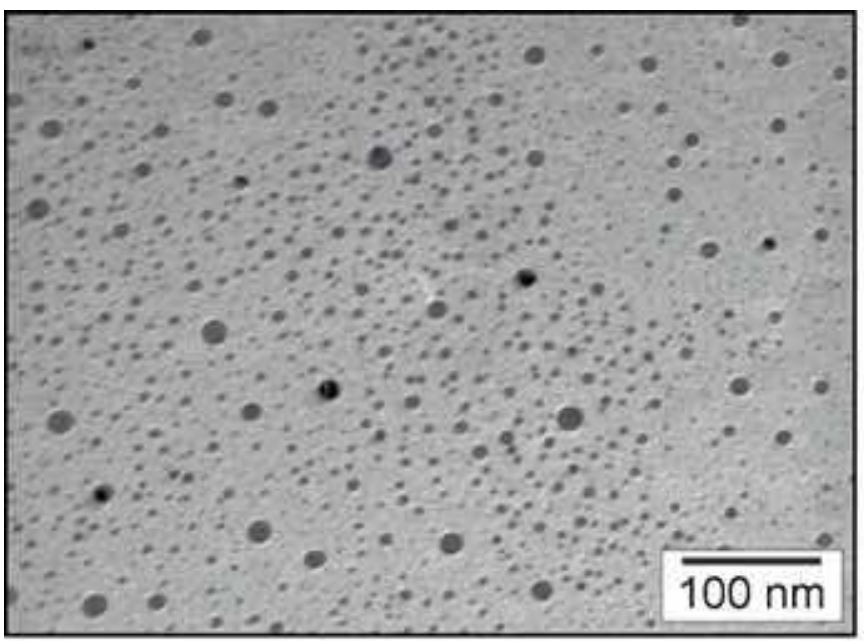

(a)

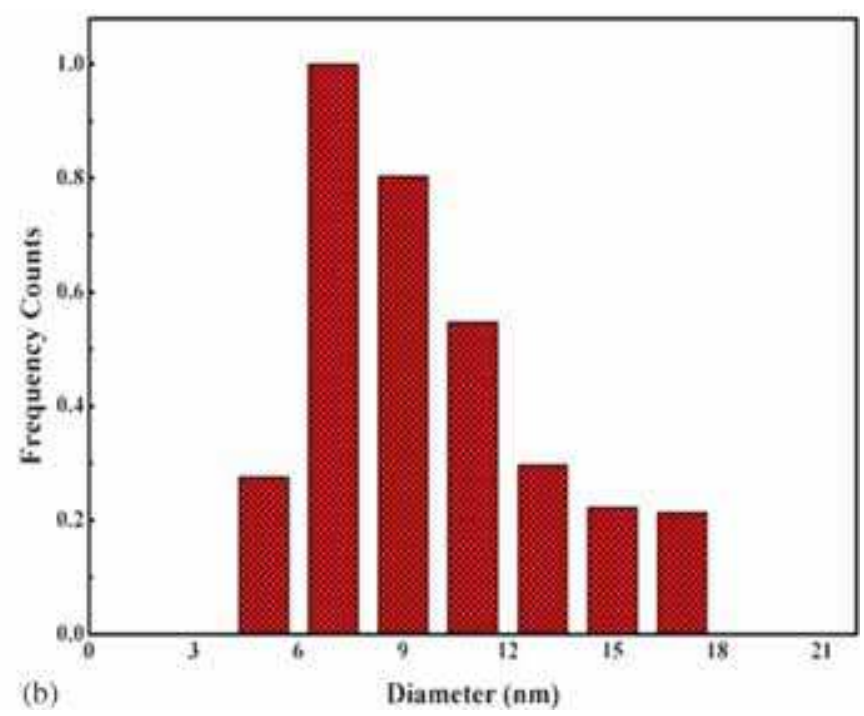

(b)
Diameter (nm)

Figure 4. (a) TEM image of SMhNPs adsorbent sample; and (b) the normalised particle size distribution found from the TEM images.

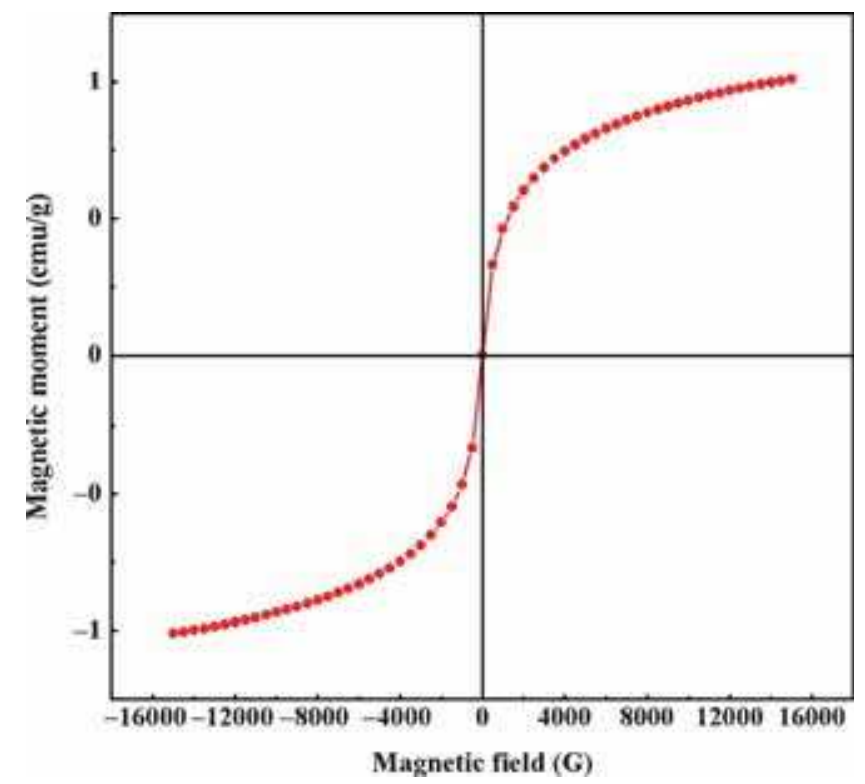

Figure 5. Plot of magnetic moment versus magnetic field for SMhNPs adsorbent sample.

in alkaline $\mathrm{pH}$. Owing to the relatively higher positive charge density at lower $\mathrm{pH}$ on the adsorbent surface, there is increased electrostatic interaction between adsorbate and adsorbent at lower $\mathrm{pH}$ which leads to increase in adsorption capacity. With increase in $\mathrm{pH}$, the adsorbent surface positive charge density decreases and consequently adsorption capacity decreases. ${ }^{29-32}$

\subsection{Effect of initial concentration on adsorption properties}

Effect of adsorbate concentration on removal efficiency by SMhNPs was studied for initial concentration range of $4-12 \mathrm{mg} \cdot \mathrm{L}^{-1}$. All the experiments for understanding the effect of initial concentration were carried out at the optimum $\mathrm{pH} 2$. Results obtained are displayed in figure 7(a). The adsorption capacity of SMhNPs increases with initial concentration of $\mathrm{Cr}(\mathrm{VI})$. The maximum experimental adsorption capacity was found to be $27.06 \mathrm{mg} . \mathrm{g}^{-1}$ when investigation was carried out with $12 \mathrm{mg} . \mathrm{L}^{-1}$ as initial $\mathrm{Cr}(\mathrm{VI})$ concentration in aqueous solution. This adsorption capacity for SMhNPs found by our group is much better than previous reports in literature on use of superparamagnetic maghemite nanoparticles as nanoadsorbents for removal of $\mathrm{Cr}(\mathrm{VI})$ from aqueous medium. To the best of our knowledge, none of the earlier studies have reported $\mathrm{Cr}(\mathrm{VI})$ adsorption capacity greater than $20 \mathrm{mg} \cdot \mathrm{g}^{-1} \cdot{ }^{33-35}$ Iron oxide nanoparticles are surface hydroxyl functionalized in aqueous medium. Such hydroxyl groups being amphoteric, at $\mathrm{pH}<\mathrm{pH}_{\mathrm{PZC}}$, the adsorbent surface is positively charged. Lower the $\mathrm{pH}$ higher is the surface charge density. On the other hand, at $\mathrm{pH}>\mathrm{pH}_{\mathrm{PZC}}$, the adsorbent will be negatively charged. We believe that starch functionalization gives additional hydroxyl groups on the adsorbent surface. ${ }^{22}$ This increases the adsorption capacity of maghemite nanoparticles by increasing the number of surface active sites, in comparison to without functionalization.

Further, the equilibrium adsorption data obtained from experiments carried out at $\mathrm{pH} 2$ and at $30^{\circ} \mathrm{C}$ for the effect of initial $\mathrm{Cr}(\mathrm{VI})$ concentration were found to fit best the Freundlich adsorption isotherm model. In contrast to the Langmuir adsorption isotherm, the Freundlich adsorption model does not assume the equality of the adsorption active sites. That is, the adsorbent surface is considered to be heterogeneous in nature. The 

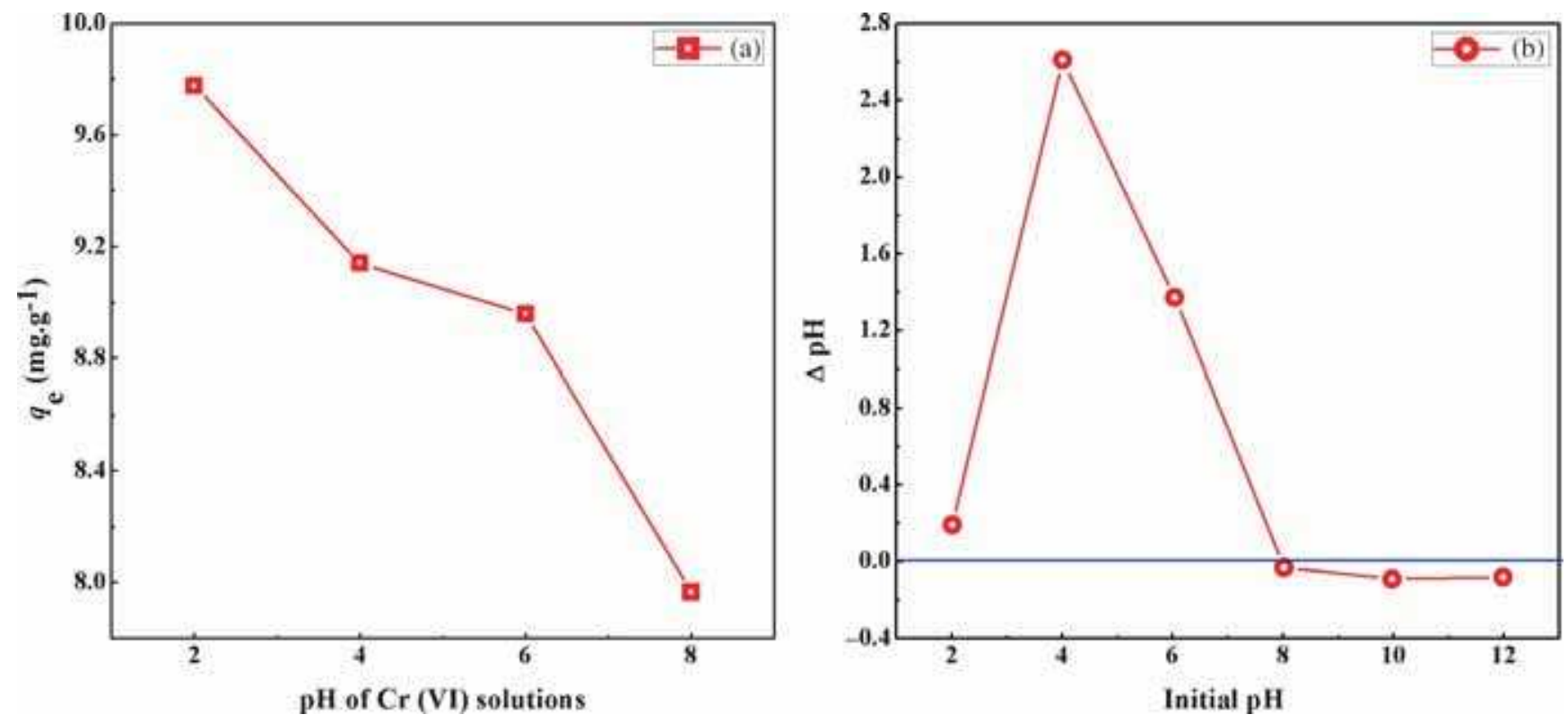

Figure 6. (a) Effect of $\mathrm{pH}$ on $\mathrm{Cr}(\mathrm{VI})$ ions removal by SMhNPs adsorbent; and (b) $\Delta \mathrm{pH}$ versus initial pH plot for the determination of the $\mathrm{pH}_{\mathrm{pzc}}$ of SMhNPs adsorbent sample. Lines joining the data points are only meant as a guide to the eye.
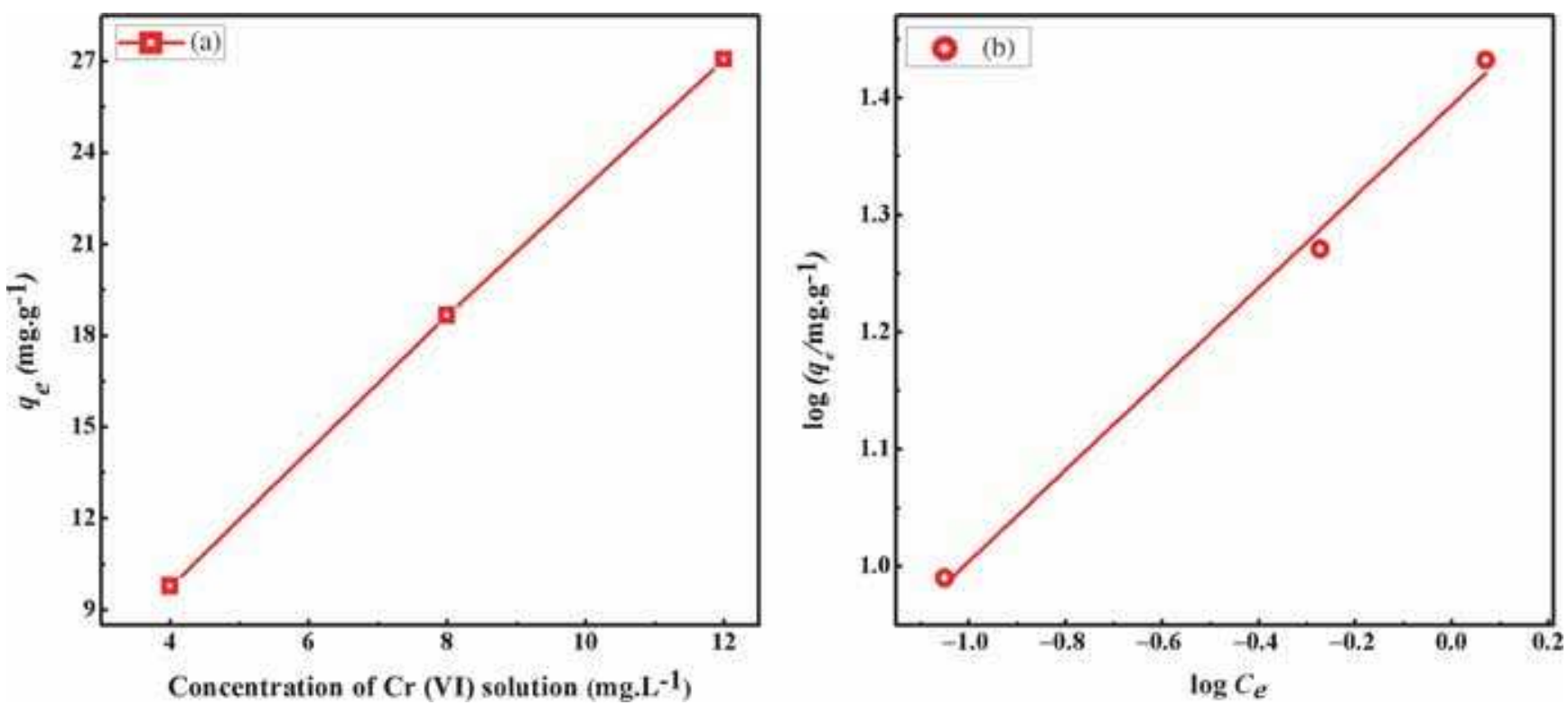

Figure 7. (a) Plot of adsorption capacity $\left(q_{e}\right)$ versus initial concentration for the removal of Cr(VI) ion by the SMhNPs adsorbent; and (b) Freundlich adsorption isotherm plot for removal of Cr(VI) ions by SMhNPs. $\mathrm{C}_{\mathrm{e}}$ is in $\mathrm{mg} \mathrm{L}^{-1}$.

graph of Freundlich adsorption isotherm of SMhNPs is given in figure 7(b). The Freundlich adsorption model follows the equation 3 .

$$
q_{e}=K_{F} C_{e}^{1 / n}
$$

Here, $q_{e}$ is the amount adsorbate of adsorbed (mg. $\mathrm{g}^{-1}$ ) on the adsorbent surface, $C_{e}$ is the adsorbate equilibrium concentration $\left(\mathrm{mg} . \mathrm{L}^{-1}\right)$ and $K_{F}$ and $1 / n$ are the Freundlich isotherm constants related to adsorption capacity and adsorption intensity respectively of adsorbents. ${ }^{36}$ The linearized form of Freundlich adsorption isotherm is given in equation 4 .

$$
\log q_{e}=\log K_{F}+\frac{1}{n} \log C_{e}
$$

The values of $K_{F}$ and $n$ were determined from the intercepts and slope of Freundlich adsorption isotherm plot of figure 7(b), respectively. The adsorption capacity $\left(K_{F}\right)$ for the removal of $\mathrm{Cr}(\mathrm{VI})$ by SMhNPs from aqueous solutions at $303 \mathrm{~K}$ and $\mathrm{pH} 2$ is found to be 
24.76 (in units of $\left.(\mathrm{mg} / \mathrm{g})(\mathrm{L} / \mathrm{mg})^{1 / \mathrm{n}}\right)$, and $1 / n$ value is 0.3895 , respectively, which suggests that the adsorption process is favorable and the adsorption capacity increases with increase of adsorption sites. Even the $K_{F}$ value indicates a much better adsorption capacity of SMhNPs adsorbent for $\mathrm{Cr}(\mathrm{VI})$ removal as compared to those reported for maghemite nanoparticles in literature. ${ }^{37-40}$

\subsection{Adsorption kinetic studies}

Experiments for determining the kinetics of adsorption of $\mathrm{Cr}(\mathrm{VI})$ onto the SMhNPs were done at $\mathrm{pH} 2$ and at $4 \mathrm{ppm}$ initial adsorbate concentration conditions. The rate of $\mathrm{Cr}(\mathrm{VI})$ removal by SMhNPs was fitted to both pseudo first order and second order rate equations. Whether the kinetics data obtained agrees better with pseudo-first or second order rate expression is determined by finding the regression coefficient of linear fit $\left(R^{2}\right)$ and non-linear fit Chi-square $\left(\chi^{2}\right)$. Whenever $R^{2}$ values for fits to different equations are very close, then the Chi-square test is helpful in identifying the best fitted adsorption rate expression. ${ }^{31} \chi^{2}$ value measures the difference between experimental adsorption capacity $\left(q_{e}(\mathrm{E})\right)$ and theoretical adsorption capacity $\left(q_{e}(\mathrm{C})\right)$. Mathematically this expression is given by equation 5 .

$$
\chi^{2}=\sum \frac{\left(q_{e}(E)-q_{e}(C)\right)^{2}}{q_{e}(C)}
$$

Thus, smaller $\chi^{2}$ value corresponds to the better fitted adsorption rate expression. Using this statistical analysis we found that the rate data fit very well the pseudo-second order rate equation 6.

$$
\frac{t}{q_{t}}=\frac{1}{k_{2} q_{e}{ }^{2}}+\frac{1}{q_{e}} t
$$

Here $k_{2}$ is the rate constant $\left(\mathrm{g} \cdot \mathrm{mg}^{-1} \mathrm{~h}^{-1}\right)$ and $k_{2} q_{e}^{2}$ (mg. $\mathrm{g}^{-1} \mathrm{~h}^{-1}$ ) is the initial adsorption rate. The fitted graph is shown in figure 8(a). Straight line plots of $t / q_{t}$ versus $t$ confirmed that the adsorption process followed second order kinetics. Table 1 shows the first and second adsorption kinetics parameter for the adsorption of $\mathrm{Cr}(\mathrm{VI})$ ions by SMhNPs. First and second order rate constants at different temperatures were obtained from the slope and intercept of corresponding graphs, respectively.
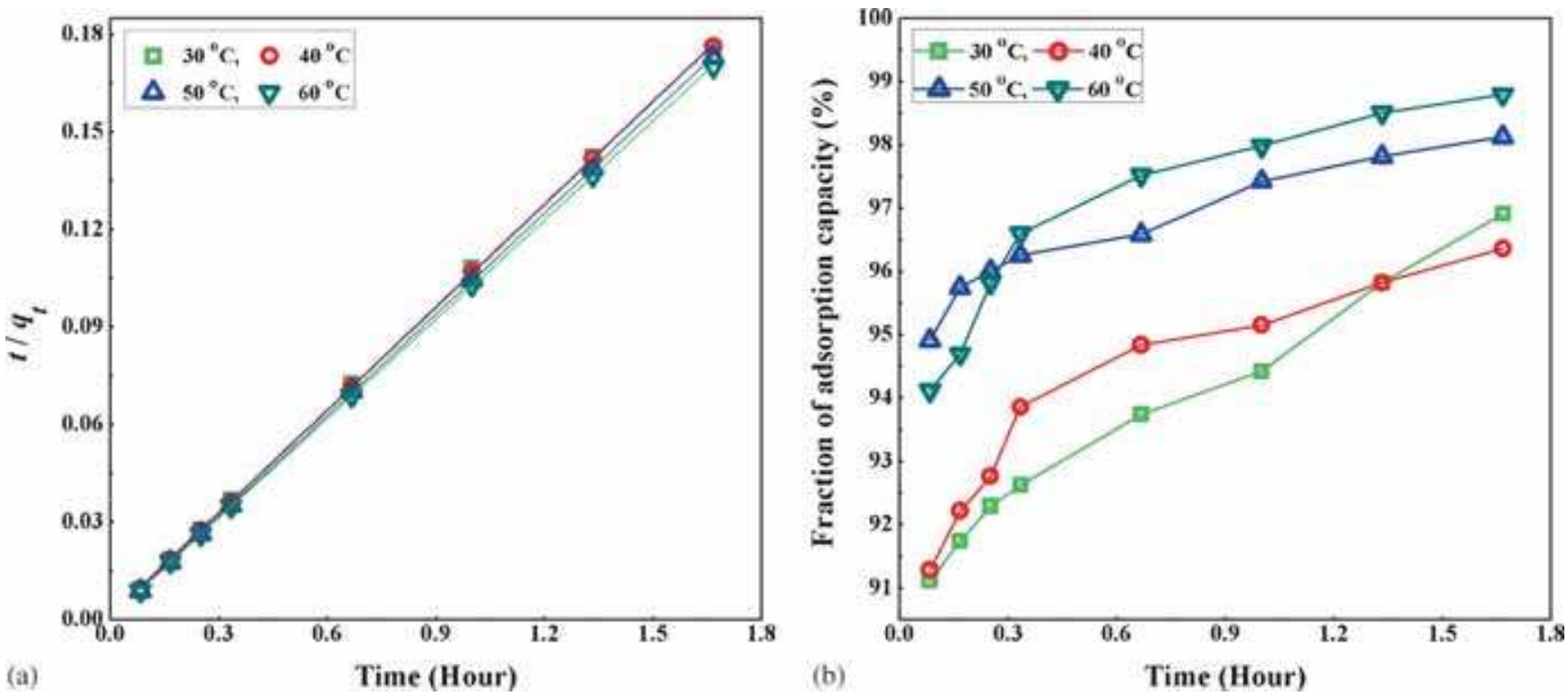

Figure 8. The pseudo-second order kinetics graph of SMhNPs adsorbent sample: (a) $t / q_{t}$ versus time (t) at different temperatures, and (b) Fraction of adsorption capacity versus time (t) at different temperatures.

\begin{tabular}{|c|c|c|c|c|c|c|c|c|}
\hline \multirow[b]{2}{*}{ Temp (K) } & \multicolumn{4}{|c|}{ Pseudo second order Kinetic model } & \multicolumn{4}{|c|}{ Pseudo first order Kinetic model } \\
\hline & $q_{e}\left(\mathrm{mg} \cdot \mathrm{g}^{-1}\right)$ & $k_{2}\left(\right.$ g. $\left.\mathrm{mg}^{-1} \mathrm{~h}^{-1}\right)$ & $R^{2}$ & $\chi^{2}$ & $q_{e}\left(\mathrm{mg} \cdot \mathrm{g}^{-1}\right)$ & $k_{2}\left(\right.$ g. $\left.\mathrm{mg}^{-1} \mathrm{~h}^{-1}\right)$ & $R^{2}$ & $\chi^{2}$ \\
\hline 303 & 9.480 & 7.673 & 0.999 & 0.009 & 0.908 & 0.615 & 0.975 & 86.618 \\
\hline 313 & 9.473 & 11.383 & 0.999 & 0.011 & 0.796 & 0.510 & 0.927 & 101.944 \\
\hline 323 & 9.647 & 16.661 & 0.999 & 0.002 & 0.473 & 0.586 & 0.970 & 184.531 \\
\hline
\end{tabular}

Table 1. Pseudo first and second order adsorption kinetics parameter for the adsorption of $\mathrm{Cr}(\mathrm{VI})$ ions by SMhNPs. 
Increase in adsorption capacity with the time is displayed in figure $8(\mathrm{~b})$. Initially the adsorption capacity increases rapidly with time. However, after the initial adsorption stage, the capacity increases more slowly. This phenomenon may be explained in the following manner. Initially vacant/active adsorption sites are readily available to adsorbate molecules. Therefore, in the initial stages, adsorption capacity increases rapidly. However, with increase in time two factors come into play. One is adsorbate-adsorbate repulsion and the second factor is lesser availability of vacant adsorbent surface active sites. Thus, after a certain period of time the adsorption capacity reaches its saturation or equilibrium value.

\subsection{Adsorption activation energy}

The activation energy for the adsorption of $\mathrm{Cr}(\mathrm{VI})$ on the surface of starch functionalized maghemite nanoparticles is calculated using Arrhenius equation. The linear form of Arrhenius adsorption activation energy expression is represented by equation 7 .

$$
k_{2}=A \exp \left(-\frac{E_{a}}{R T}\right)
$$

Equation 8 gives the logarithmic form of Arrhenius adsorption activation energy.

$$
\ln k_{2}=\ln A-\left(\frac{E_{a}}{R T}\right)
$$

Here, $k_{2}$ is the adsorption rate constant at solution temperature $T$ (in $\mathrm{K}$ ), $A$ is the pre-exponential factor (in $\left.\mathrm{g} . \mathrm{mg}^{-1} \cdot \mathrm{h}^{-1}\right), E_{a}$ is the adsorption activation energy (in $\mathrm{kJ}^{-\mathrm{mol}^{-1}}$ ) and $R$ is universal gas constant $\left(8.314 \mathrm{~J} \cdot \mathrm{mol}^{-1} \cdot \mathrm{K}^{-1}\right)$. Adsorption rate constant for the adsorbate on the surface adsorbent were calculated from the pseudo-second order rate equation. Plot of $\ln$ $\mathrm{k}$ versus $1 / \mathrm{T}$ showed a very good linear fit. Adsorption activation energy is then calculated from the negative slope $\left(E_{a} / R\right)$ of this plot, shown in figure 9 . The numerical value of adsorption activation energy is commonly used to differentiate between physisorption and chemsorption. Physical adsorption processes are reversible in nature, rapidly attain equilibrium and also have smaller adsorption activation energy e.g., ranging from $5-40 \mathrm{~kJ} . \mathrm{mole}^{-1}$. Alternatively, chemisorption is specific and requires larger adsorption activation energies (40 to $800 \mathrm{~kJ} \mathrm{~mol}^{-1}$ ) due to chemical bond formation between adsorbate and adsorbent species. ${ }^{41-45}$ The activation energy for the adsorption of $\mathrm{Cr}(\mathrm{VI})$ on the surface of SMhNPs has been found to be $31.54 \mathrm{~kJ} \mathrm{~mol}^{-1}$ which suggests physical adsorption of $\mathrm{Cr}(\mathrm{VI})$.

\subsection{Desorption study}

From the results of the previous sections, it was found that for $\mathrm{Cr}(\mathrm{VI})$ removal SMhNPs have low adsorption activation energy and follow the Freundlich adsorption isotherm model. This indicates that the removal of $\mathrm{Cr}(\mathrm{VI})$ ions onto SMhNPs should be reversible. Therefore, the possibility of regeneration of the adsorbent and its reuse was investigated. The first step in this process involves adsorbent regeneration and recovery of the component adsorbed. ${ }^{46-48}$ The desorption of $\mathrm{Cr}(\mathrm{VI})$ from SMhNPs was achieved by shaking Cr(VI) loaded SMhNPs adsorbent sample in $0.1 \mathrm{M} \mathrm{NaOH}$ solution for 24 hours at $298 \mathrm{~K}$. At the end of this process SMhNPs were washed thoroughly with distilled water until no more $\mathrm{Cr}(\mathrm{VI})$ could be detected in the washings and the filtrate also became neutral. It is worth mentioning that more than $80 \%$ of the SMhNPs could be recovered after washing. The recovered adsorbent was reused. This process of adsorbent recovery and reuse was done for five consecutive cycles. Although some amount of SMhNPs was always lost during recovery, we took care to keep the amount of reused adsorbent constant in repeat cycles. This was achieved by carrying out several parallel adsorption-desorption experiments. That is,

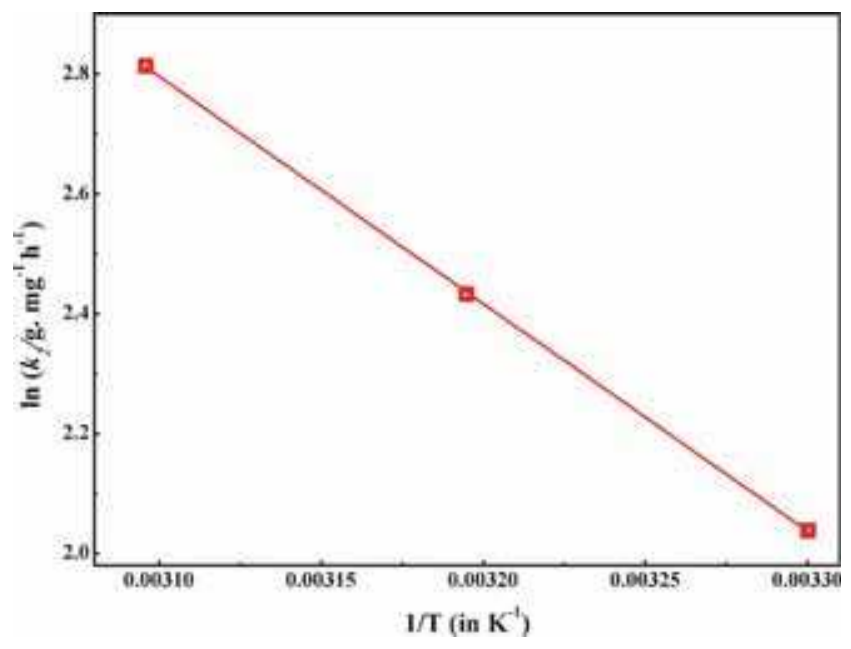

Figure 9. $\ln k_{2}$ versus $1 / T$ plot for the determination of the adsorption activation energy.

Table 2. Percentage change in adsorption capacity $\left(q_{e}\right)$ with desorption cycles at $12 \mathrm{ppm}$.

\begin{tabular}{lcc}
\hline Desorption cycle & $q_{e}\left(\mathrm{mg} \cdot \mathrm{g}^{-1}\right)$ & $\%$ change in $q_{e}\left(\mathrm{mg} \cdot \mathrm{g}^{-1}\right)$ \\
\hline 1 & 27.065 & 0.002 \\
2 & 26.833 & 0.857 \\
3 & 25.996 & 3.951 \\
4 & 25.124 & 7.170 \\
5 & 24.295 & 10.234 \\
\hline
\end{tabular}


Table 3. Thermodynamic parameters for the adsorption of $\mathrm{Cr}(\mathrm{VI})$ ions by SMhNPs adsorbent at different temperatures (K).

\begin{tabular}{lcccc}
\hline Temperature $(K)$ & $q_{e}\left(\mathrm{mg} \cdot \mathrm{g}^{-1}\right)$ & $\Delta G^{o}\left(\mathrm{~kJ} . \mathrm{mol}^{-1)}\right.$ & $\Delta H^{o}\left(\mathrm{~kJ} \cdot \mathrm{mol}^{-1}\right)$ & $\Delta S^{o}\left(\mathrm{~J} . \mathrm{mol}^{-1} \cdot \mathrm{K}^{-1}\right)$ \\
\hline 303 & 9.776 & -9.521 & 78.848 & 291.648 \\
313 & 9.808 & -10.248 & 84.052 & 308.825 \\
323 & 9.816 & -10.690 & 89.423 & 326.551 \\
\hline
\end{tabular}

adsorbent loss was compensated from parallel experiments. Table 2 shows variation of adsorption capacity against desorption cycles. We observe that decrease in adsorption capacity is almost negligible till the second cycle. From third cycle onwards 3-4\% loss in adsorption capacity was observed in every cycle. Therefore, we may conclude that SMhNPs show good recovery and reuse properties with respect to $\mathrm{Cr}(\mathrm{VI})$ removal.

\subsection{Adsorption thermodynamic studies}

Adsorption thermodynamic parameters such as change in standard Gibb's free energy $\left(\Delta G^{o}\right)$, enthalpy $\left(\Delta H^{o}\right)$, entropy $\left(\Delta S^{o}\right)$ for adsorption of $\mathrm{Cr}(\mathrm{VI})$ by starch functionalized maghemite nano-adsorption were calculated using equations 9-11.

$$
\begin{gathered}
\Delta G^{0}=-R T \ln K \\
\Delta H^{0}=-\ln \left(\frac{K_{1}}{K_{2}}\right)+R\left(\frac{T_{2} T_{1}}{T_{2}-T_{1}}\right) \\
\Delta S^{0}=\frac{\Delta H^{0}-\Delta G^{0}}{T}
\end{gathered}
$$

Here, $K$ is the adsorption equilibrium constant, $R$ is ideal gas constant and $T$ is temperature. Table 3 displays the values of $\Delta G^{o}, \Delta H^{o}$ and $\Delta S^{o}$ for the adsorption of $\mathrm{Cr}(\mathrm{VI})$ by SMhNPs. It is found that $\Delta G^{o}$ becomes more negative with increase in temperature from 30 to $60^{\circ} \mathrm{C}$, which indicates the spontaneous nature of adsorption. The $\Delta H^{o}$ value is positive and increases with temperature indicating that adsorption of $\mathrm{Cr}(\mathrm{VI})$ on the surface of SMhNPs is endothermic. Consequently $\Delta S^{o}$ value is also positive and increases with temperature. This suggests increase in disorder in between adsorbent-adsorbate interface with increase in temperature. The adsorption capacity of SMhNPs for $\mathrm{Cr}(\mathrm{VI})$ with the temperature of the adsorbate solution shows very small increase $(\sim 1.5 \%)$ as temperature (table 3) is increased from 30 to $60^{\circ} \mathrm{C}$. This increase may be due to creation of new adsorption surface active sites at higher temperatures by the breakage of new bonds.

\section{Conclusions}

Starch functionalized maghemite nanoparticles were synthesized by a co-precipitation method for the removal of $\mathrm{Cr}(\mathrm{VI})$ ions from aqueous solutions. Adsorption is highly $\mathrm{pH}$ dependent and adsorption capacity decreases with increase in $\mathrm{pH}$. Maximum or optimum $\mathrm{Cr}(\mathrm{VI})$ removal occurs at $\mathrm{pH} 2$. Experiments at increasing initial $\mathrm{Cr}(\mathrm{VI})$ concentration (at the optimum $\mathrm{pH}$ ) reveal significant improvement in maximum adsorption capacity when compared with earlier literature reports on maghemite nanoparticles. We believe that this is a consequence of increase in access to functional groups afforded by starch on the surface of nano-adsorbents. Adsorption experimental data agree with the Freundlich adsorption isotherm model and adsorption activation energy is also in agreement with the physical adsorption mechanism. The adsorption kinetics follows the pseudo-second order rate equation. Thermodynamic investigations demonstrated that the adsorption of $\mathrm{Cr}(\mathrm{VI})$ on the surface of SMhNPs is spontaneous, endothermic and adsorption efficiency increases with temperature.

\section{Acknowledgements}

Authors wish to express their sincere thanks to SAIF, Indian Institute of Technology Madras, Chennai, for Vibrating sample magnetometer (VSM) characterization, Department of Anatomy, Institute of Medical Sciences for Transmission electron microscopy (TEM) characterization and Department of Nanoscience Banaras Hindu University for using UV-Visible instrument. P. N. Singh is thankful to the University Grant Commission, Government of India, for the financial support under the senior research fellowship Scheme (SRF).

\section{References}

1. Ouki S K and Neufeld R D 1997 J. Chem. Technol. Biotechnol. 703

2. Alvarez-Ayuso E, Garcia-Sanchez A and Querol X 2003 Water Res. 374855

3. Dupont L and Guillon E 2003 Environ. Sci. Technol. 37 4235 
4. Gupta V K, Agarwal S and Saleh T A 2011 Water Res. 452207

5. Wei C, German S, Basak S and Rajeshwar K 1993 J. Electrochem. Soc. 14060

6. Huijing Q, Yanjun W U, Yong L I U and Xinhua X U 2008 Front. Environ. Sci. Engin. China 251

7. Singh V K and Tiwari P N 1997 J. Chem. Technol. Biotechnol. 69376

8. Lazaridis N K and Asouhidou D D 2003 Water Res. 37 2875

9. Gupta V K, Mohan D, Sharma S and Park K T 1999 The Environmentalist 19129

10. Gupta V K, Gupta M and Sharma S 2001 Water Res. 35 1125

11. Gupta V K and Ali I 2004 J. Colloid. Interface. Sci. 271 321

12. Ali I and Gupta V K 2007 Nat. Protoc. 12661

13. Gupta V K and Rastogi A 2008 J. Hazard. Mater. 154 347

14. Gupta V K and Rastogi A 2009 J. Hazard. Mater. 163 396

15. Singh A K, Gupta V K and Gupta B 2007 Anal. Chim. Acta 585171

16. Hu F Q, Wei L, Zhou Z, Ran Y L, Li Z and Gao M Y 2006 Adv. Mater. 182553

17. Bertholon I, Hommel H, Labarre D and Vauthier C 2006 Langmuir 225485

18. Alibeigi S and Vaezi M R 2008 Chem. Eng. Technol. 31 1591

19. Likhitkar S and Bajpai A K 2012 Carbohydr. Polym. 87 300

20. Hu J, Chen G and Lo I M C 2005 Water Res. 394528

21. Kim D K, Mikhaylova M, Wang F H, Kehr J, Bjelke B, Zhang Y, Tsakalakos T and Muhammed M 2003 Chem. Mater. 154343

22. Singh P N, Tiwary D and Sinha I 2014 J. Environ. Chem. Eng. 22252

23. Hu J, Chen G, Lo I M C and Asce M 2006 J. Environ. Eng. 132709

24. Bajpai A K and Shrivastava J 2005 Polym. Int. 541524

25. Shen Y F, Tang J, Nie Z H, Wang Y D, Ren Y and Zuo L 2009 Sep. Purif. Technol. 68312
26. de Faria D L A, Venaüncio Silva S and de Oliveira M T 1997 J. Raman Spectrosc. 28873

27. Slavov L, Abrashev M V, Merodiiska T, Gelev Ch, Vandenberghe R E, Markova-Deneva I and Nedkov I 2010 J. Magn. Magn. Mater. 3221904

28. Kizil R, Irudayaraj J and Seetharaman K 2002 J. Agric. Food Chem. 503912

29. Tuutijärvi T, Lu J, Sillanpää M and Chen G 2009 J. Hazard. Mater. 1661415

30. Hu J, Lo I M C and Chen G 2007 Sep. Purif. Technol. 56249

31. Boparai H K, Joseph M and O'Carroll D M $2011 \mathrm{~J}$. Hazard. Mater. 186458

32. Sarkar S, Dutta S, Bairi P and Pal T 2014 Langmuir 30 7833

33. Jiang W, Pelaez M, Dionysiou D D, Entezari M H, Tsoutsou D and O'Shea K 2013 Chem. Eng. J. 222527

34. Wang P and Lo I M C 2009 Water Res. 433727

35. Hu J, Lo I M C and Chen G 2007 Sep. Purif. Technol. 5876

36. Li Y, Gao B, Wu T, Sun D, Li X, Wang B and Lu F 2009 Water Res. 433067

37. Pino G H, de Mesquita L M S and Torem M L 2006 Sep. Sci. Technol. 413141

38. Banerjee S S, Joshi M V and Jayaram R V 2004 Sep. Sci. Technol. 391611

39. Bishnoi N R, Bajaj M, Sharma N and Gupta A 2004 Bioresour. Technol. 91305

40. Nameni M, Alavi Moghadam M R and Arami M 2008 Int. J. Environ. Sci. Tech. 5161

41. Zhang D, Ma Y, Fenga H and Hao Y 2012 J. Chil. Chem. Soc. 57964

42. Dogan M and Alkan M 2003 Chemosphere 50517

43. Malkoc E and Nuhoglu Y 2007 Sep. Purif. Technol. 54 291

44. Zhang W B, Deng M, Sun C X and Wang S B 2014 Ind. Eng. Chem. Res. $\mathbf{5 3} 333$

45. Ho Y S and Mckay G 1998 Chem. Eng. J. 70115

46. Hu J, Chen G and Lo I M C 2005 Water Res. 394528

47. Lasheen M R, El-Sherif I Y, Sabry D Y, El-Wakeel S T and El-Shahat M F 2014 Desalin. Water Treat. 526464

48. Hu J, Lo I M C and Chen G 2005 Langmuir 2111173 